\title{
Transferability of driver speed and lateral deviation measurable performance from semi-dynamic driving simulator to real traffic conditions
}

\author{
Maria Gemou
}

Received: 1 April 2013 / Accepted: 28 May 2013 / Published online: 4 July 2013

(C) The Author(s) 2013. This article is published with open access at SpringerLink.com

\begin{abstract}
Purpose This manuscript presents the experimental plan and results of driver trials conducted with a semi-dynamic driving simulator and an equipped research vehicle in real traffic conditions.

Methods 12 trainees, 12 novice (driving license less than 1.5 years; 25,000 totally run km by average) and 12 experienced drivers (driving license more than 12 years; 320,000 totally run $\mathrm{km}$ and $28583 \mathrm{~km}$ only the last year by average) participated in the trials, conducting the same driving sessions in the simulator and on-the-road, consisting of a free driving and a following vehicle scenario in the context of highways, rural and urban roads. Their maximum speed, difference of their average speed from each speed limit and their average lateral deviation in driving simulator and onthe-road were compared. A novel research framework was established for the results analysis dealing with the existence of numerical proximity between the driving simulator and the on-the-road measurements (in the sense of absolute validity), the degree of this proximity and the possibility and the type of the correction that could be applied leading to an acceptable absolute validity.

Results Derived results have shown that several degrees of numerical proximity apply for different combinations of driver behaviour metrics, driver cohorts, driving tasks and road contexts, whereas the type of the driver behaviour metric seems to be the most determining factor for absolute validity existence.

Conclusions Tangible findings consist of transfer algorithms that serve as the basis for redefining warning and intervention thresholds of ADAS but also driver training and assessment schemes based on driving simulators.
\end{abstract}

M. Gemou $(\bowtie)$

Centre for Research and Technology Hellas-Hellenic Institute of Transport, Egialias 52, Marousi 15125, Athens, Greece

e-mail: mgemou@certh.gr
Keywords Transferability of driving simulator results . Driving simulator validity $\cdot$ Experimental plan $\cdot$ Speed . Lateral deviation $\cdot$ Conversion matrix

\section{Introduction}

The multiple benefits of driving simulation and the variety of its application in several fields is responsible for the increase of their popularity the last decade. Driving simulators are definitely offering flexibility, cost efficiency and elimination of traffic risk in comparison to real traffic conditions, whether their use is being scheduled in the context of research or drivers' training and assessment. Next to the recognised benefits of using driving simulators, a series of restrictions associated to them, dealing with simulator sickness and driver overconfidence issues, lack of sufficient realism, etc. not rarely raise concerns regarding the usefulness and effectiveness of their use and their main scope, being the sufficient reflection of reality, is quite often ambiguous.

The most critical characteristics that determine the reliability of a driving simulator, with respect to the degree up to which it achieves to represent reality, are considered to be its technical fidelity (or physical validity) and its behavioural validity $[1,2]$, with the behavioural validity being considered even more important, due to its applicability to specific research hypotheses and the fact that in many cases high technical fidelity is not synonymous to maximum reliability in terms of results.

While there are several types of behavioural validity, absolute and relative validity of driving simulators are the most frequent ones being investigated. The simulator has absolute validity with regard to a research question if the absolute size of the effect is comparable to the absolute size of the effect in reality, whereas it has relative validity with regard to this question, if, for instance, the direction or relative size of the measure is the same as in reality [3]. 
There is a series of research studies tackling with driving simulators behavioural validity and different types of it (i.e. relative validity, absolute validity, construct validity, etc.), in the context of which it was intended to investigate the relationship of driving simulator measurements with those coming from real traffic conditions. Among other researchers, Blana [4] was one of the first ones that, in 1996, reviewed and compared the most driving behavioural research studies concerning driving simulator validity being conducted until that moment, reaching finally the conclusion that the type of validity reached is independent to the technical fidelity, type and cost of the driving simulator, which is in agreement with previous argumentation of Evans [24] and other researchers.

More recent studies followed [5-10], each one studying the results of comparative trials in simulator and on-road across different driver behaviour metrics and road safety measures and in several road contexts and along various driving tasks/scenarios. In most cases, relative validity has been proved, but this was not necessarily the case for absolute validity.

Although the numerical sizes are usually different, and as such, absolute validity cannot be claimed, this has not hindered a series of researchers to claim that the drivers behaviour in simulator is in general similar to their on-road behaviour [5, 11-13]. Some researchers $[14,15]$ have also claimed that driving simulators may serve in order to identify the most "dangerous" drivers and those that are likely to be involved in collisions [16].

While the unproved absolute validity might not be a hinder for cases where the tendency and the effect size of the results may matter the most (i.e. when road safety measures are under investigation), there are cases that numerical proximity is critical, such as in the cases that the warning or intervention thresholds of an Advanced Driver Assistance System (ADAS) need to be determined.

Also, it is a fact that the outcomes of different studies are always bounded to the specific research question, while, quite often, even for very similar research questions, the outcomes vary significantly, which reveals the weakness in translating the derived results with consistency to real world conditions. As such, prior to the real study that would safely utilise the driving simulator results in order to conclude about any road safety issue, the adjusted to the specific research hypotheses investigation of the validity of each driving simulator is necessary, while, the often vague results, do not allow safe extrapolations to real traffic conditions.

As such, and in order to maximise the proved benefits of driving simulators in the most cost-effective way and avoid the duplication of trials in real traffic conditions, when it is not necessary, there is an emerging need to overcome the aforementioned weaknesses, through an approach that would allow the most reliable and most accurate possible transferability of driving simulator results to real traffic conditions, independent of the specific research hypotheses as well as the technical characteristics of the driving simulator.

In other words, it is important to know in advance which is the a priori relationship of the driving behaviour in driving simulator and in real traffic conditions and if there is such one, regardless of the research question being investigated each time, so that the primary and most important "translation" of the measurements of the driving simulator to real traffic conditions is feasible. In this way, not only the in advance more valid interpretation of the experimental results of the driving simulator, but also the further recognition of the specific gravity of a specific research hypothesis will be allowed.

Such a knowledge would minimise the need for a former study of the driving simulator validity and an afterwards study for verification of the results in real traffic conditions, which, in turn, would imply minimization of the research/education resources with the in parallel availability of valid results. In addition, in this way, the technical adjustment of the driving simulator in order to approach as much as possible as a technical means the real traffic context will be no longer necessary.

This need has been underlined from various researchers, some of which have investigated several approaches for the design of driving simulator that would export measurements close to reality [17-21].

However, so far, no fundamental approach has been established [22, 23], in order to allow driving simulators to be safely used as reliable substitutes of reality.

Riener (2010) [10] underlines that in order to be able to use the driving simulator in place of a real vehicle for trials with users, it should be ensured that we are having meaningful results that will be directly transferable to real traffic conditions, proposing two solutions. The first one is the use of very high fidelity simulators, which are usually expensive (even for rent) and which do not necessarily lead to reliable results [24-27] and this is the reason that no further effort should be invested in this direction, especially in specific application areas like driver training $[25,26]$.

The second solution proposed by Riener [10] is the availability of a conversion matrix/model that would provide for each simulator fidelity level, the correction required for each participating parameter, so that the driving simulator measurements approximate as much as possible the respective on-the-road results.

In order to respond to the above recognized need, a research framework has been developed, being reflected in the research hypotheses described in Section 2 of this manuscript. In order to accommodate the acceptance or rejection of those research hypotheses, experimental trials were planned aiming to gather driving performance data from a driving simulator and real traffic conditions through a specially equipped vehicle. The 
experimental plan is being discussed in Section 3 of this manuscript. Section 4 discusses the methodology followed for the analysis of the results, and which sets the basis for the novel approach being developed, whereas Section 5 provides indicative results from the analysis of the experimental data. Section 6 summarises the main conclusions and the steps proposed for further research with respect to this work.

This manuscript discusses only an excerpt of the overall research framework and methodology being developed as well as of the overall derived results. It specifically discusses the results concerning the drivers' maximum speed, the difference of their average speed from the speed limit and their average lateral deviation.

\section{Research Hypotheses}

There were 6 research hypotheses being established in total. The purpose was to develop research hypotheses that would be as simple as possible, lacking specific measures. In this way, the results' extrapolation would be the maximum possible, since the results that would enable the research hypotheses acceptance or rejection would serve as baseline data for future research or even for reconsideration of past research.

Research hypothesis 1: For some driving behaviour metrics, some driving tasks and some driver cohorts, there is an acceptable numeric proximity of the driving behaviour in each type of simulator and real traffic conditions; as such, no further correction is required (in the sense of absolute validity).

Research hypothesis 2: For some driving behaviour metrics, some driving tasks and some driver cohorts, there is no acceptable numeric proximity of the driving behaviour in each type of simulator and real traffic conditions in advance; however, the same direction is being observed between the numerical values of the driving simulator and those corresponding to real traffic conditions (meaning that their difference is either positive or negative for the whole population), which, after the necessary correction following the linear model, results in an acceptable numeric proximity of the respective values, allowing the extraction of safe conclusions from the driving simulator measurements concerning the real traffic conditions (in the sense of absolute validity).

Research hypothesis 3: For some driving behaviour metrics, some driving tasks and some driver cohorts, there is neither an acceptable numeric proximity of the driving behaviour in each type of simulator and real traffic conditions in advance nor the same direction in the measurements (in the sense that is being explained above), however there is a non-linear correlation, sufficiently strong, which results in an acceptable numeric proximity of the respective values, allowing the extraction of safe conclusions from the driving simulator measurements concerning the real traffic conditions (in the sense of absolute validity).

Research hypothesis 4: For some driving behaviour metrics, some driving tasks and some driver cohorts, no numerical proximity, but, instead, a qualitative proximity is being reached (i.e. all measurements from driving simulator and real traffic conditions are either under or above a specific defined threshold, after correction or not; in the sense of relative validity).

Research hypothesis 5: For some driving behaviour metrics, some driving tasks and some driver cohorts, no acceptable numeric or qualitative proximity is concluded between each type of simulator and real traffic conditions, after correction or not, linear or non-linear, and, as such, only the real traffic conditions measurements should be taken into consideration (no absolute or relative validity is being obtained).

Research hypothesis 6: The behaviour of drivers belonging to specific cohorts presents bigger proximity (numeric or qualitative) between simulator and real traffic conditions, in some specific experimental conditions. In the same way, bigger correlation is being noticed in driving behaviour for some specific types of roads, types of scenarios or driving behaviour parameters in comparison to others, at least in some specific experimental conditions.

The current manuscript deals specifically with those research hypotheses that investigate the existence-and under which conditions-of the absolute validity of the driving simulator, meaning in this case the numerical proximity of the measurements collected regarding the drivers' speed and lateral deviation in simulator and real traffic conditions, after correction or not. These are namely research hypotheses $1,2,3$ and 5 , as being presented above.

\section{Experimental Plan}

\subsection{Scope of the Trials}

In order to accommodate the acceptance or rejection of the research hypotheses of the previous section for a semidynamic driving simulator, experimental trials were planned aiming to gather driving performance data from the drivers while driving in the driving simulator and on-the-road.

It was necessary to enable the gathering of comparable driving behaviour data, meaning data able to describe the behaviour of each driver with respect to major driving behaviour metrics in simulator and on-road, in the same road 
and driving task context in each case, so that the only variable would be the driving context (simulation environment vs. real traffic conditions).

In order to serve this scope, trials ought to be conducted with those drivers groups, and in such road and driving contexts that would be the most adequate ones for this comparative study. In this sense, the comparative driving behaviour needed to be investigated in relation to those driving behaviour metricssame ones in the driving simulator and real traffic conditionsthat would be able to reveal essential findings.

\subsection{Experimental Plan Variables}

One of the most critical tasks in the construction of the experimental plan has been the identification of the appropriate variables that would satisfy the scope of the defined research hypotheses. The independent variable of the experiment was by default the context of driving (driving simulator vs. road). A study has been conducted for defining the cofounding variables of the experiment. According to it, these were determined as follows:

1. Driving experience of the subjects, which has proved to be the driver characteristic that is most likely responsible for differentiating the driving behaviour between real traffic conditions and in simulator in comparison to others (i.e. gender, age), mainly due to the driver predictive mental models that are being progressively developed as long as driving experience increases, and, due to which, driving behaviour or novice drivers is expected to be less differentiated (between the driving simulator and the real traffic conditions) in comparison to experienced drivers [28]. As such, 36 drivers were equally clustered in three levels of driving experience. As such, 12 trainees, 12 novice drivers and 12 experienced drivers participated in the trials. A driver was considered trainee, when having no driving license yet. Novice drivers were considered those with driving experience less than 3 years and less than $75000 \mathrm{veh} / \mathrm{km}$ in total, whereas experienced drivers were considered those with driving experience more than 3 years and more than $75000 \mathrm{veh} / \mathrm{km}$ in total (and at least $15,000 \mathrm{veh} / \mathrm{km}$ the last year).

Trainees and novice drivers were distinguished in two clusters, as trainees constitute the main driver cohort that is being addressed in driving skills evaluation field. Experienced drivers, on the other hand, are those that most frequently selected to participate in research regarding the evaluation of Advanced Driver Assistance Systems (ADAS) in comparison to novice drivers who, being more familiar with the new technologies, tend to be overconfident and trust excessively in it. Elderly drivers were not selected in the cluster of the experienced drivers, due to the fact that they require higher fidelity driving simulators in comparison to young or middle aged drivers (field of view of $120-180^{\circ}$ is required), whereas above 50 years old, their susceptibility to simulator sickness is significantly increasing [29]. Also, in the biggest age range, age is linearly correlated to driving experience [30], whereas differentiations that are solely related to age begin after the age of 60-65 years old [30-34]. Therefore, and since it was difficult to recruit elderly inexperienced drivers, age of the drivers was not selected to be a separate cofounding variable for this experiment.

Finally, there is no sufficient evidence that gender is factor of the differentiation of the driving behaviour in driving simulators and real traffic conditions, especially in the middle ages [30, 35]. Also, socioeconomic and geographic factors have been obliterated, since the subjects have been recruited from the same country and of similar socioeconomic status. In addition, all subjects were selected to be novice users of the driving simulator (since prior experience with the driving simulator would likely be a cause for differentiations in driving).

2. Driving task/road scenario: Two different driving tasks/road scenarios have been selected as the most applicable for the selected research hypotheses, which are namely the free driving scenario and the following vehicle scenario. The following vehicle driving task has been selected because it has been recognized as a major road safety issue [30,36, 38], mainly due to its close relation to the often catastrophic rear-end collisions, while it is important that both driving tasks, due to them being quite generic, lacking specific measures imposition, are optimum for allowing the collection of comparative data which would serve as horizontal "exposure data" for other, more sophisticated driving tasks/scenarios.

3. Road context: Three major road types have been selected, namely the urban context, the rural context and the highway context, being the major road contexts being mandatorily present in all driver educational schemes, and for which different Intelligent Transportation Systems (ITS) apply.

\subsection{Driving Behaviour Metrics}

The dependent variables have been the driver performance results in the driving simulator and on-road, across a series of driver behaviour metrics which were selected to be the most applicable ones according to the following criteria:

1. The driving behaviour metric has to be measurable in both contexts, meaning the trials with a research vehicle in real traffic conditions and in trails with a driving simulator.

2. The metric has to be as much as possible representative of the driving behaviour and adequate to express or sufficiently correlate with road safety. 
3. The metric has to be directly or indirectly extracted from the log systems of the two means-the driving simulator and the research vehicle-and in a way as to minimise as much as possible the logging errors (met quite often in the research vehicles logging mechanisms).

4. The nature of the metric has to relate with/be applicable for all the selected driving tasks/road scenarios.

The maximum vehicle speed, the difference of the average vehicle speed from the speed limit (different for each type of road), the average and maximum lateral deviation, the average and minimum headway as well as the probabilities of Time Exposed Headway (TEHP*) and of Time Integrated Headway $\left(\text { TIHP* }^{1}\right)^{1}$ were the metrics that have been selected according to the above criteria. The current manuscript focuses in specific on the maximum vehicle speed, the difference of the average vehicle speed from the speed limit (different for each type of road) and the average lateral deviation.

In specific, vehicle speed has been one of the most important safety influencing metrics [39] and perhaps the indicator which is most often met in relevant studies. Average speed, speed variance and maximum speed are the most common indicators being measured [40]. However, instead of the average speed, the difference of the average vehicle speed from the speed limit was selected to be measured, due to the fact that constitutes a key safety indicator for the Intelligent Speed Adaptation (ISA) systems. In specific, we chose to measure the difference of the average speed from the speed limit instead of the difference of the maximum speed of the speed limit, as the first is more able to reveal the permanent critical driving behaviour in comparison to the second one (i.e. a driver may outreach the speed limit occasionally when reaching his/her maximum speed, but it is definitely more important if his/her average speed is critical in relation to the speed limit). Also, the speed variance has not been selected to be measured, as when being measured in free or stable driving conditions (like in our case; see below), does not result in sufficiently transparent conclusions [40]. Therefore, the difference of the average vehicle speed from the speed limit and Time Headway (THW)-the latest not being analysed in the current manuscript-were measured instead in the context of this experiment.

In addition, although Time-to-Line Crossing (TLC) is considered to be a useful indicator in driving simulator trials, it is often difficult to measure it in real traffic conditions without a specially equipped research vehicle [41]. Also, if the lane markings do not match with the safe track of the vehicle, as this is perceived by the driver, invalid and difficult to interpret results are emerging [42]. Therefore, the

\footnotetext{
${ }^{1}$ Adjustment of TETP* and TITP* metrics respectively, as these have been introduced by [37], where Time to Collision has been replaced by Time Headway.
}

average and the maximum lateral deviation were selected to be measured instead. The maximum lateral deviation was measured in addition to the average lateral deviation, because the later often leads to misleading results (i.e. a driver who often follows a straight track may have the same average lateral deviation with someone that systemically deviates from his/her track). Nevertheless, for the measurement of the lateral deviation, high definition sensors and constant lane width are required. Also, it was chosen to measure the average lateral deviation instead of the standard deviation of lateral deviation (which is being proposed by several researchers), since due to the relatively small differentiations, the standard deviation of lateral position is significantly related with the duration of the data [40], which is not long enough in our case.

\subsection{Apparatus}

For the conduct of the trials, a semi-dynamic driving simulator (SMART cabin prototype) and a specially equipped research vehicle (Lancia Thesis $2.420 \mathrm{~V}$ Emblema) have been used (see Fig. 1). This equipment belongs to the Hellenic Institute of Transport of the Centre for Research and Technology Hellas (http://www.hit.certh.gr).

The position of all control levers of the driving simulator (windshield wipers, blinker, ignition key and light switch) is transmitted to the driving computer. All operational elements, steering wheel, accelerator pedal, brake pedal, gearshift lever and handbrake lever, provide nature-true force reactions. The gearshift functions like in the real car either as automatic or "soft tip" with incrementing and decrementing the six gears and with reverse gear.

The sight system includes five large monitors each having a width of $2 \mathrm{~m}$ and 500 field of vision. There is on-screen projection with consumer video projectors with 2,500 ANSIlumen. The sound system generates original sounds according to the situation (starter, engine noise, horn, screeching of tires, drive wind, rain, etc.). The vibration device creates nature true vibrations of the car according to the revolution of the simulated engine. The simulator is equipped with specialised software which allows the development of special driving scenarios, according to the purposes of each study.

The research vehicle is equipped with several sensors as it can be seen in Fig. 1. The collected information from the sensorial system is being transmitted from the vehicle electronic system to the central Personal Computer (PC) for storage and further processing.

\subsection{Experimental Process}

Each of the 36 drivers participating in the trials executed the two driving tasks/scenarios (free driving and following vehicle) in the three road contexts (highway, rural, urban) in each, in the driving simulator and in real traffic conditions with the 
Fig. 1 HIT research equipment (left: research semi-dynamic driving simulator; right: equipped research vehicle)
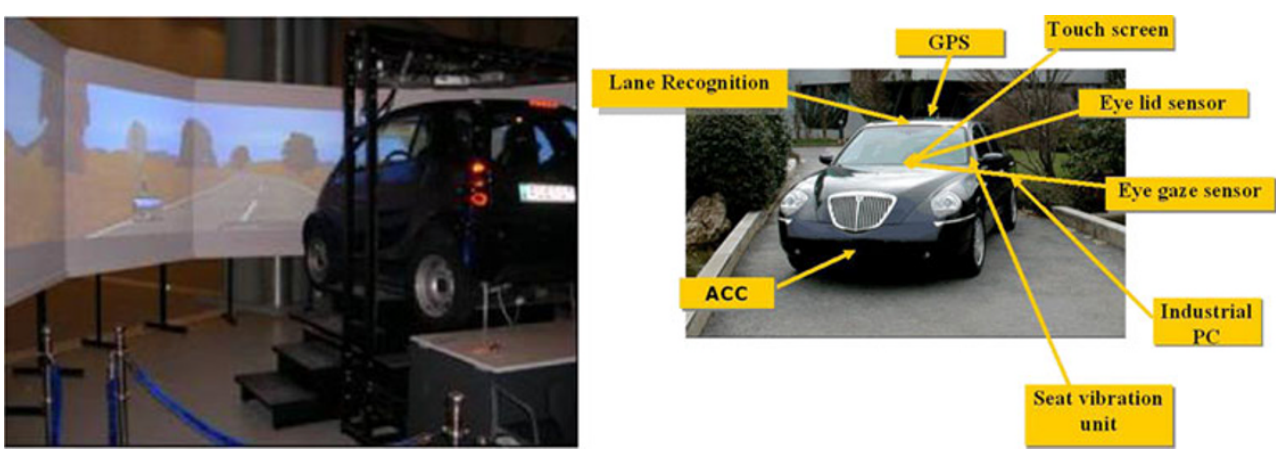

equipped research vehicle. For learning effects elimination, half of the drivers started with the execution of the scenarios in the driving simulator and continued with the real traffic conditions, whereas the other half of them followed the reverse way.

Prior to the realisation of the trials, the supervisors introduced the participants to the objective of the trails and the process that would be followed, also handed to them in printed form. It should be highlighted that the guidelines provided to each participant were the same. Each participant signed an informed consent for and completed a form aiming to gather demographic data. An awareness session of the participants with the research equipment of about $15 \mathrm{~min}$ followed (with no logging).

In addition to the trials supervisor who was present in all trials realised, a driving instructor was present in the vehicle during the trials conducted in real traffic conditions. After the completion of the driving sessions, each participant completed a questionnaire which concerned his/her perception of the driving simulator realism, a questionnaire concerning the simulator sickness symptoms-Simulator Sickness Questionnaire [43], a Rating Scale of Mental Effort [44], aiming to collect participants views on the mental effort required during driving in the driving simulator and a Driving Quality Scale [45], aiming to collect participants views on how good they drove in relation to their average driving in real traffic conditions.

Driving scenarios and contexts were initially designed and completed in real traffic conditions, and their closest possible representation followed in the driving simulator. The other road users in the driving simulator scenarios were given "smart" attributes, whereas the main traffic characteristic that was paid attention to was the traffic density (medium traffic density was selected in all cases). Environmental conditions were also chosen to be similar in simulation and reality conditions. Lack of rain and snowfall and similar lighting conditions were ensured. All the above conditions was a matter of design in the driving simulator, whereas in real traffic conditions it was a matter of careful time of day and day of week selection for the trials conduct.

The two scenarios (free driving and following vehicle) were executed successively by each driver and the same was valid for the different road contexts in each scenario (highway, rural, urban). The duration of each sub-session (in each type of road in each scenario) was about $5 \mathrm{~min}$ in both the driving simulator and real traffic conditions.
Highway roads had 2 traffic lanes per direction with a parapet and an emergency traffic lane, whereas rural and urban roads had one traffic lane per direction.

In the free driving scenario, the driver was asked to drive free and as safely as possible according to his/her opinion, paying attention so as to remain in the right traffic lane (i.e. not executing frequent lane changes). The average speed of the other road users (being observed in real traffic conditions and respectively designed in the driving simulator) was $40 \mathrm{~km} / \mathrm{h}$ in urban roads, $60 \mathrm{~km} / \mathrm{h}$ in rural roads and $100 \mathrm{~km} / \mathrm{h}$ in highways.

In the following vehicle scenario, the driver was asked to follow a vehicle driving in front of him/her maintain the safest possible distance, according to his/her opinion. The condition for following vehicle situation is when $\mathrm{THW} \leq 5 \mathrm{~s}$. $[46,47]$. The respective condition for "cautious" driving has been selected to be THW $\leq 3 \mathrm{~s}$. and for "risky" driving when THW $\leq 1,5 \mathrm{~s}$. [47]. The lead vehicle was driven by a driving instructor and drove with steady speed in the right traffic lane (forcing in this way the ego vehicle to move also in the right lane). The average speed of the lead vehicle and the other road users was $35 \mathrm{~km} / \mathrm{h}$ in the urban roads, $60 \mathrm{~km} / \mathrm{h}$ in the rural roads and $100 \mathrm{~km} / \mathrm{h}$ in highways.

Another instruction that was given to the drivers in both scenarios was to reach the minimum suggested speed and then try to maintain it as his/her minimum one. The reason for this restriction was that that there was a relevant restriction in the driving simulator software (a minimum speed has to be reported, above which logging occurs) and because it was considered necessary to set some minimum speeds, representative of each traffic environment, that would minimise irrelevant $\operatorname{logs}$ that would constitute "noise" in the logged data (i.e. related with unexpected stations, etc.). The minimum speeds suggested for each scenario are as follows:

Free driving scenario

- Urban context: $28 \mathrm{~km} / \mathrm{h}$

- Rural context: $50 \mathrm{~km} / \mathrm{h}$

- Highway context: $80 \mathrm{~km} / \mathrm{h}$

Following vehicle scenario

- Urban context: $28 \mathrm{~km} / \mathrm{h}$

- Rural context: $50 \mathrm{~km} / \mathrm{h}$ 
- Highway context: $90 \mathrm{~km} / \mathrm{h}$

The trials supervisors were given the guideline to monitor the above minimum speeds and advice/remind the participants accordingly.

\section{Methodology for Analysis}

After the processing of the results, the comparative analysis of the data collected from the trials in the driving simulator and the trials in the real traffic conditions followed. For the analysis of the specific variables, the following conditions were defined, which negotiate the sense of the "accepted proximity" of the above presented research hypotheses, with or without correction, in the context of the absolute validity. These are as follows:

1. Correlation Type A: «Numerical proximity of original driving simulator measurements to real traffic conditions measurements»»

- Condition: When the following condition is valid for each individual driver (and, consequently, for all drivers belonging to the same group):

$$
\begin{aligned}
& \text { Deviation } \\
& =\mid\left(\text { Value }_{\mathrm{N} \_ \text {simulator }}-\text { Value }_{\mathrm{N} \_ \text {vehicle }}\right) / \text { Value }_{\mathrm{N} \_ \text {vehicle }} \mid \leq 0,15
\end{aligned}
$$

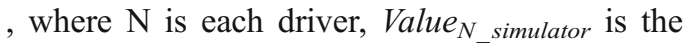
value of the measurement in driving simulator and Value $_{N_{-} \text {vehicle }}$ is the value of the measurement for the same driver in real traffic conditions. In the above equation, by "Deviation", the absolute deviation is meant.

In this case, we are interested in the numerical proximity of the values for each driver (in simulator and real traffic conditions), which is being expressed through the size of their absolute deviation.

In other words, the absolute deviation of the numerical values (of each metric under investigation) must be less or equal to $15 \%$ for each driver. Consequently, the mean absolute deviation of all drivers belonging to the same group (always consisting of 12 drivers) must be less or equal to $15 \%$.

The set value of 0,15 (or $15 \%$ given in percentage) has been selected due to the maximum possible error of the vehicle sensorial system measurements, which varies between $12 \%-15 \%$ (depending the measurements conditions and the road context), which sets a tolerance threshold.
The reference value in the estimation of the absolute deviation is the driver performance (in each metric) in real traffic conditions, since, any correlation and correction of the respective simulator measurement will occur with respect to it.

- Transfer algorithm: In this case, we assume that the values of driving simulator and the real traffic conditions are correlated by default through the lineal model $\mathrm{y}=\mathrm{ax}+\mathrm{b}$, where $\mathbf{a}=\mathbf{1}$ and $\mathbf{b}=\mathbf{0}$.

- Practical meaning: The results following this condition imply that no further correction is required in the driving simulator measurements.

2. Correlation Type B: «Relative numerical proximity of original driving simulator measurements to real traffic conditions measurements»)

- Condition 1: The absolute deviation between driving simulator and real traffic condition is less or equal to $15 \%$ for at least the $75 \%$ of the drivers (belonging to the same driver group).

\section{Deviation}

$$
=\mid\left(\text { Value }_{\mathrm{N}_{\mathrm{N}} \text { simulator }}-\text { Value }_{\mathrm{N} \_ \text {vehicle }}\right) / \text { Value }_{\mathrm{N} \_ \text {vehicle }} \mid \leq 0,15,
$$

for at least $75 \%$ of the drivers (belonging to the same driver group).

- Condition 2: The corresponding mean absolution deviation of all drivers (belonging to the same driver group) is less or equal to $20 \%$ (15\% for $100 \%$ of the drivers is rationally equal to $20 \%$ for the $75 \%$ of the drivers).

As in the previous condition, also in this condition, we are interested in the numerical proximity of the measurements for each driver (in driving simulator and in real traffic conditions), which is being expressed through the size of their absolute deviation, for at least the $75 \%$ of the drivers (following the $75^{\text {th }}$ percentile).

- Transfer algorithm: In this case, we assume that the correlation of the measurements values in the driving simulator and real traffic conditions is being expressed by default, for at least $75 \%$, by the lineal model $\mathbf{y}=\mathbf{a x}+\mathbf{b}$, where $\mathbf{a}=\mathbf{1}^{*}$ and $\mathbf{b}=\mathbf{0}$.

- Practical meaning: The results following this condition imply that no further correction is required of the driving simulator measurements, although with some reservations (therefore, the coefficient $1^{*}$ is being used).

3. Correlation Type C: "Relative numerical proximity of original driving simulator measurements to real traffic 
conditions measurements, after correction following the linear model":

- Condition 1: When at least one of the two conditions of Correlation Type B is not valid.

- Condition 2: When for at least the $75 \%$ of the drivers of each driver group, the same direction is being observed between the numerical values of the driving simulator and those corresponding to real traffic conditions (meaning that their difference is either positive or negative for all the $75 \%$ of the drivers):

$$
\text { Value }_{N_{\text {_vehicle }}} \leq \text { Value }_{\mathrm{N} \_ \text {simulator }}
$$

or:

Value $_{\text {N_vehicle }} \geq$ Value $_{\text {N_simulator }}$

- Condition 3: When, after the correction of the original driving simulator measurements, the numerical proximity conditions of Correlation Type A or Correlation Type B are being fulfilled.

- Transfer algorithm: In this case, we assume that the measurement values from the driving simulator and the real traffic conditions are correlated by the linear model, $y=a x+b$, where $a$ and $b$ coefficients can be estimated with the following two ways:

1. Through the mean absolute difference (of the values of the driving simulator and those corresponding to real traffic conditions): in this case, a is symbolically equal to $\widetilde{1}^{*}$ (symbol expresses mean value) and $b$ is equal to the mean absolute difference (of the values of the driving simulator and those corresponding to real traffic conditions).

2. Through linear regression: in this case, $a$ and $b$ are occurring after applying linear regression. However, this method has been rejected, due to being sensitive to the specific sample serving as control sample.

Thus, in this case, having estimated coefficient a and $b$ (actually $b$, since $a$ is always the same), and following the first correction way, since the $2^{\text {nd }}$ one has been rejected, correction of the original driving simulator measurements is following, in order to investigate the numerical proximity of the corrected driving simulator measurements with the real traffic conditions measurements (Condition 3).

- Practical meaning: The results complying with this condition imply that there is no original numerical proximity, but, due to the same sign direction of the differences between the measurements (for at least the $75 \%$ of the population), and in order for the intended numerical proximity to occur, a flat correction may be applied in the driving simulator measurements.

4. Correlation Type D: «Relative numerical proximity of original driving simulator measurements to real traffic conditions measurements, after correction following nonlinear models»»

- Condition: None of the previous Correlation Types is being fulfilled.

- Transfer algorithm: In this case, we investigate the possibility of correcting the driving simulator measurements using curve fitting.

- Practical meaning: Numerical proximity is resulted, after correction following the non-linear models.

5. Correlation type E: "No correlation of any type that results in numerical proximity of results, with or without correction"

- Condition: None of the previous Correlation Types is being fulfilled.

- Practical meaning: No valid correction way, resulting in satisfactory numerical proximity of measurements is being recognized.

Correlation Type D has been rejected, due to being sensitive to the specific sample serving as control sample. As such, whenever Correlation Types A, B and C are not occurring, then Correlation Type D-E is directly implied.

The 36 drivers' performance results concerning maximum vehicle speed, on the difference of the average vehicle speed from the speed limit, and the average lateral deviation were being logged in the respective software of the driving simulator and the research vehicle. Before the analysis of the results, a post processing of the collected measurements were being done. The processing that concerned the speed and the lateral deviation measurements was related to the elimination of the "noisy" data, as well as the translation to the correct SI (Le Système International d'unités) units. Also, during the processing, a tolerance of $85 \%$ was considered concerning the suggested minimum speeds, meaning that, regardless the guideline that was given to the drivers, speeds that were $15 \%$ below the respective guideline for each road context and driving scenario were set in the driving simulator and become acceptable.

In addition, all measurements in the following vehicle scenario were filtered according to the following vehicle condition aforementioned (THW $\leq 5$ s.). All measurements that were not in agreement with this condition were being erased.

Finally, in both simulator and research vehicle recording systems, the position of the vehicle in each case is originally given in relation to the edge of the lane.However, in order to have a steady reference point (which cannot be the continuously changing lane width of the real traffic lanes), an after processing tool place in 
order to translate the recorded distances from the edge of the lane to the distances from the middle of the drivers' lane.

The automation of the processing was made feasible by respective software that was developed in Java.

\section{Indicative Results}

\subsection{Sample Demographics}

An overview of the participants demographics is given in the following table (Table 1). None of the trainees reported previous experience with the driving simulator. Only one novice driver and 2 experienced drivers had previous experience with the driving simulator (less than an hour).

\subsection{Results \& Discussion}

\subsubsection{Maximum Speed}

The maximum speed of the drivers was analysed only for the free driving scenario, since in the following vehicle scenario, the drivers were instructed to follow the lead vehicle which moved with a steady speed, and, as such, we expected no significant findings from the observation of their maximum speed.

The most important findings that emerged from the comparative analysis of the maximum speed of the drivers are as follows:

- The maximum speed of the trainees and the experienced drivers in highway and urban roads present relative numeric proximity, with no further need for correction (Correlation Type B; see Fig. 2).
- On the contrary, the maximum speed of novice drivers in highway and urban roads presents accepted numeric proximity, only after correction (Correlation Type C). It should be highlighted that the correction is of the same size ("-5") (see Fig. 3 series). The maximum speed of the trainees in rural road presents also accepted numeric proximity after correction (Correlation Type C), which is smaller than above ("-3,5") (see Fig. 3 series).

- In all other cases, there is no accepted numeric proximity emerging (Correlation Type D-E).

- It is worth noting that, in each case, when a correction was necessary (when a Correlation Type C occurred), the maximum speed in the driving simulator was always bigger than the one in real traffic conditions.

According to the cross-check of the results with the conditions as being presented in Section 5, the relevant transfer algorithms have been constructed.

As it has been explained in Section 5, the Correlation Types A, B and C, are following the linear model $\mathrm{y}=\mathrm{ax}+\mathrm{b}$. Correlation Types A and B are following the simplified model, $\mathrm{y}=\mathrm{ax}$, whereas Correlation Type $\mathrm{C}$ includes also coefficient $b$, which is equal to the mean absolute difference, whereas its sign is positive or negative, according to the dominating one-way correlation of the numeric values of the driving simulator measurements and the measurements of the research vehicle in real traffic conditions. In the case of the speed, the values of coefficient $b$ have been rounded in the closest first decimal (i.e. 1-1,5-2-2,5...).

Coefficient a is given the values $1,1^{*}$ and $\widetilde{1}^{*}$, according to the Correlation Type (A, B or $\mathrm{C}$ respectively). It should be reminded, that whenever a Correlation Type D-E has emerged (as shown in the above table), there is no transfer algorithm applicable.

Table 1 Sample demographics

\begin{tabular}{|c|c|c|c|c|}
\hline $\begin{array}{l}\text { Number of subjects } \\
\text { per driver group }\end{array}$ & Mean driving experience & Mean age & $\begin{array}{l}\text { Number } / \% \text { of } \\
\text { men-women }\end{array}$ & $\begin{array}{l}\text { Distance } \\
\text { travelled in } \mathrm{km}\end{array}$ \\
\hline 12 trainees & Without driving license & 24 years old $(\mu=23.83, \sigma=9.15)$ & $\begin{array}{l}9 \text { men-3women } \\
(75 \%-25 \%)\end{array}$ & - \\
\hline $\begin{array}{l}12 \text { novice } \\
\text { drivers }\end{array}$ & $\begin{array}{c}\text { Owing a driving license less than } \\
1.5 \text { years }\left(\mu^{\mathrm{a}}=1.16, \sigma^{\mathrm{b}}=0.71\right)\end{array}$ & 22 years old $(\mu=21,67, \sigma=3,89)$ & $\begin{array}{l}7 \text { men-5 women } \\
(58.33 \%-41.67 \%)\end{array}$ & $\begin{array}{l}\text { Totally: } \mu=25,000, \\
\begin{array}{c}\sigma=23525.61 \\
\text { The last year: }\end{array} \\
\begin{array}{c}\mu=10916.67 \\
\sigma=10219.93\end{array}\end{array}$ \\
\hline $\begin{array}{l}12 \text { experienced } \\
\text { drivers }\end{array}$ & $\begin{array}{l}\text { Owing a driving license more than } \\
12 \text { years }(\mu=12.17, \sigma=7.64)\end{array}$ & $\begin{array}{c}36 \text { years old }(\mu=35.58, \sigma=9.56) \\
\text { Maximum age: } 48 \text { years old }\end{array}$ & $\begin{array}{l}8 \text { men-4women } \\
\quad(66.66 \%-33.34 \%) .\end{array}$ & $\begin{array}{c}\text { Totally: } \mu=320,000, \\
\begin{array}{c}\sigma=246613.43 \\
\text { The last year: } \\
\mu=28583.33, \\
\sigma=17593.69\end{array}\end{array}$ \\
\hline
\end{tabular}

${ }^{\mathrm{a}} \mu=$ Mean value

${ }^{\mathrm{b}} \sigma=$ Standard deviation 

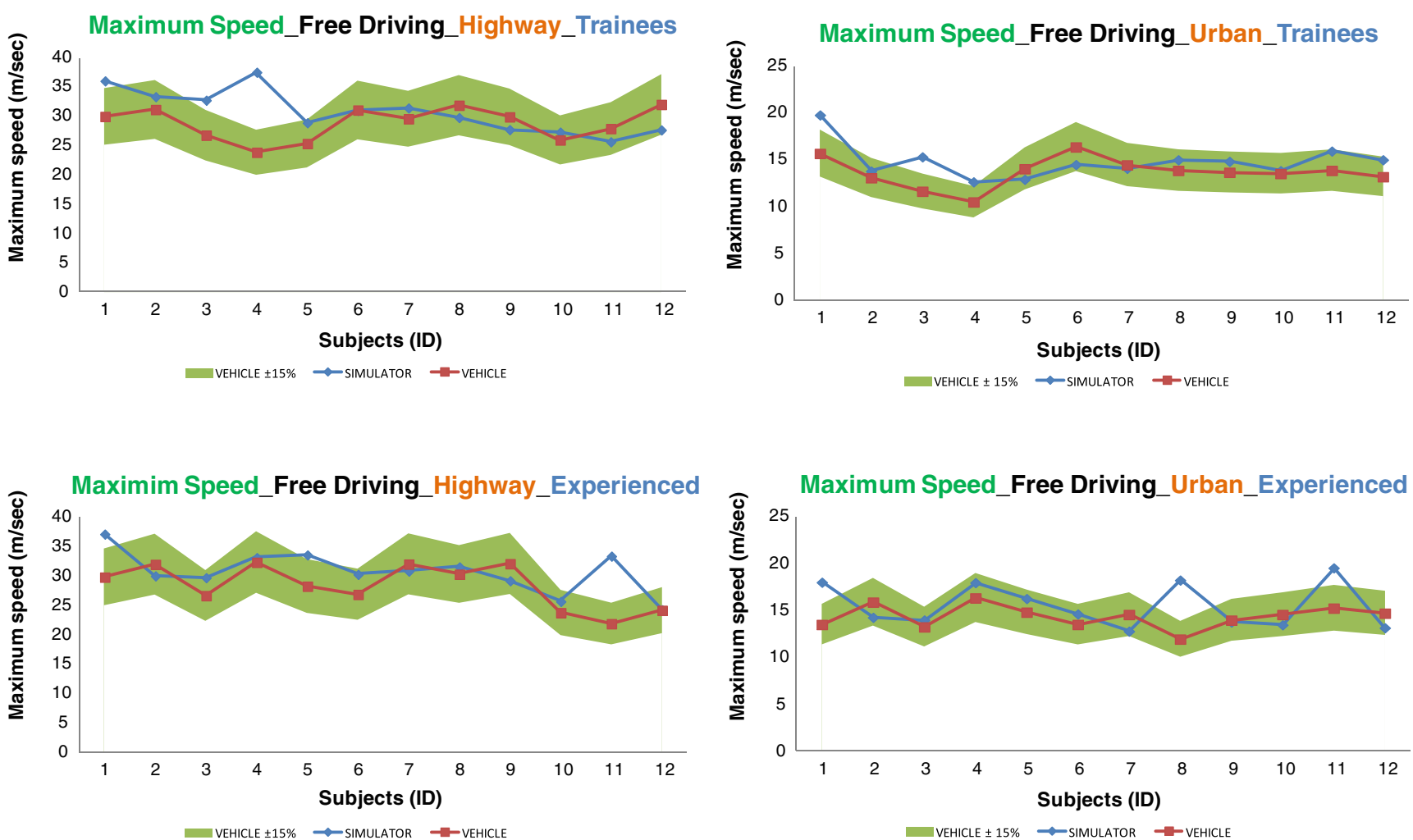

Fig. 2 Maximum speed results corresponding to Correlation Type B

Following the linear model, the relationship of speed in real traffic conditions $\left(\mathrm{V}_{\mathrm{veh}}\right)$ with speed in the simulated environment $\left(\mathrm{V}_{\text {sim }}\right)$, is given by the following equation:

$V_{v e h}=a V_{\text {sim }}+b$

If we represent the values of the $a$ and $b$ coefficients by the respective matrices (column vectors) A and $\mathrm{B}$, the above is as follows:

$V_{\text {veh }}=A V_{\text {sim }}+B$

The following matrix summarises all the transfer algorithms related to maximum speed and difference of the average speed from the speed limit (being discussed in the following section).

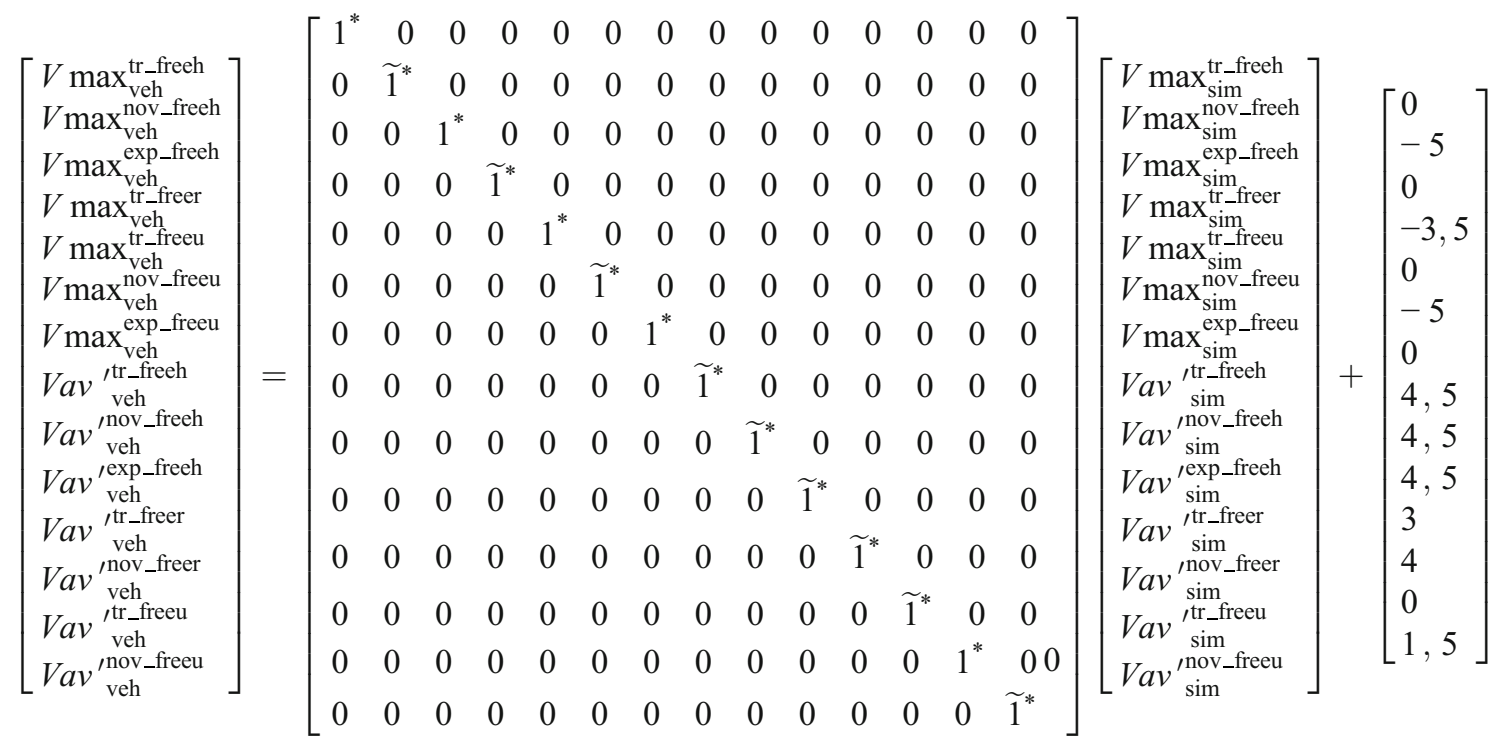


Fig. 3 Maximum speed results corresponding to Correlation Type B
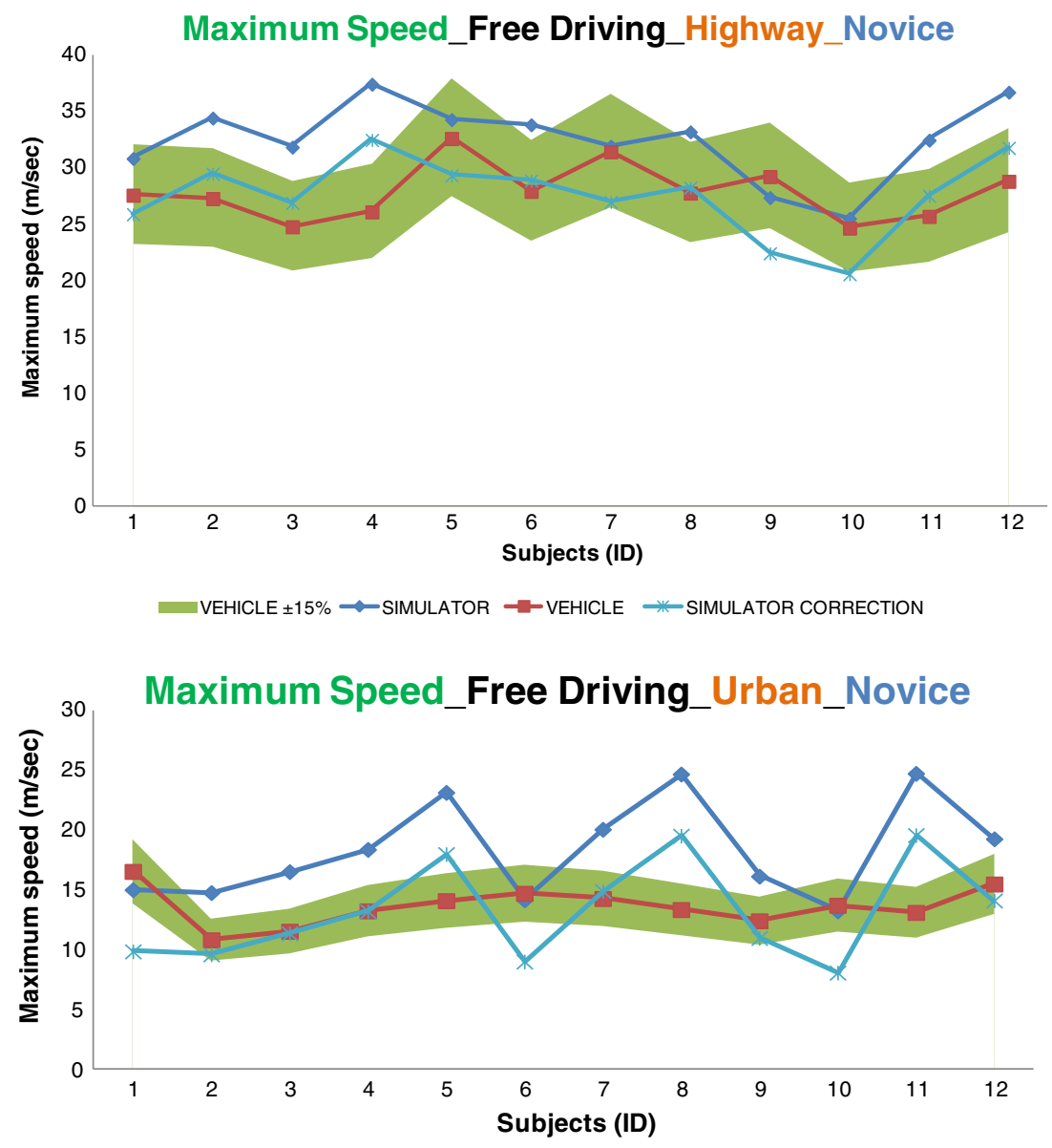

VEHICLE $\pm 15 \% \multimap$ SIMULATOR -VEHICLE $\%$ SIMULATOR CORRECTION

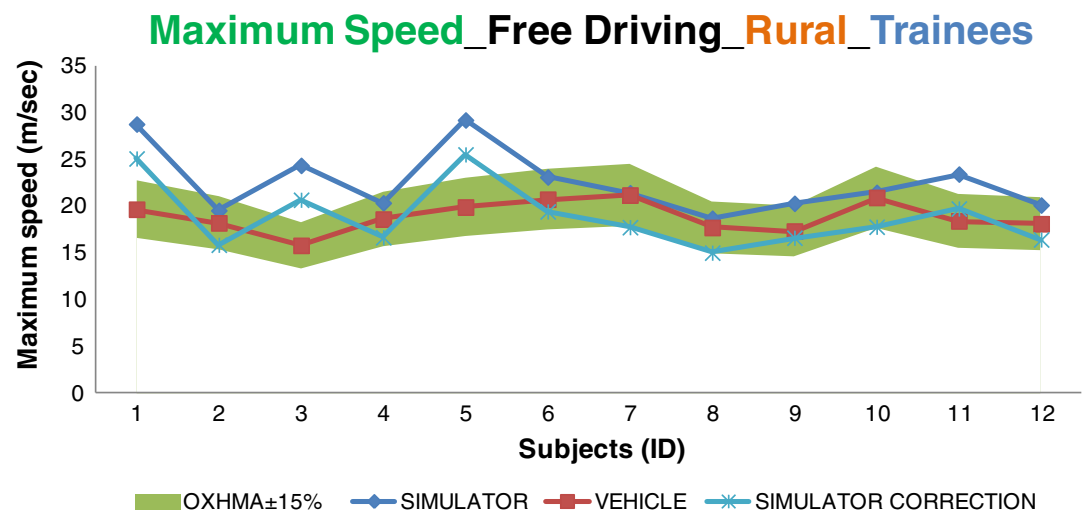

In the above matrix, "tr" refers to the trainees, "nov" refers to the novice, "exp" refers to the experts, "veh" refers to the respective measurement in real traffic conditions and "sim" to the respective measurement in driving simulator. "Vmax" refers to the maximum speed, whereas "Vav" to the difference of the average speed from the speed limit. "freeh" refers to the free driving scenario in highway, "freer" refers to the free driving scenario in rural roads and "freeu" refers to the free driving scenario in urban roads.

\subsubsection{Difference of Average Speed from Speed Limit}

The Greek speed limits for each road context are as follows:

- Highways speed limit: $130 \mathrm{~km}$

- Rural roads speed limit: $90 \mathrm{~km}$

- Urban roads speed limit: $50 \mathrm{~km}$

As in the case of maximum speed, also in this case and for the same reasons, the difference of the average speed from 
the applicable in each case speed limit was analysed only for the free driving scenario. The most important findings are as follows:

- The difference of the average speed from the speed limit of trainees, novice and experienced drivers in highway presents acceptable numerical proximity after correction (Correlation Type C). It is remarkable that in all cases, correction of the same size has emerged (" $+4,5$ "). Numerical proximity after correction is being resulted also in the case of the trainees and novice drivers in rural road (Correlation Type C). Also, in this case, the correction size is similar (" +3 and " +4 " respectively). The same is valid in the case of novice drivers in urban roads (correction is " +1.5 " in this case). See Fig. 4 for results corresponding to Correlation Type C.
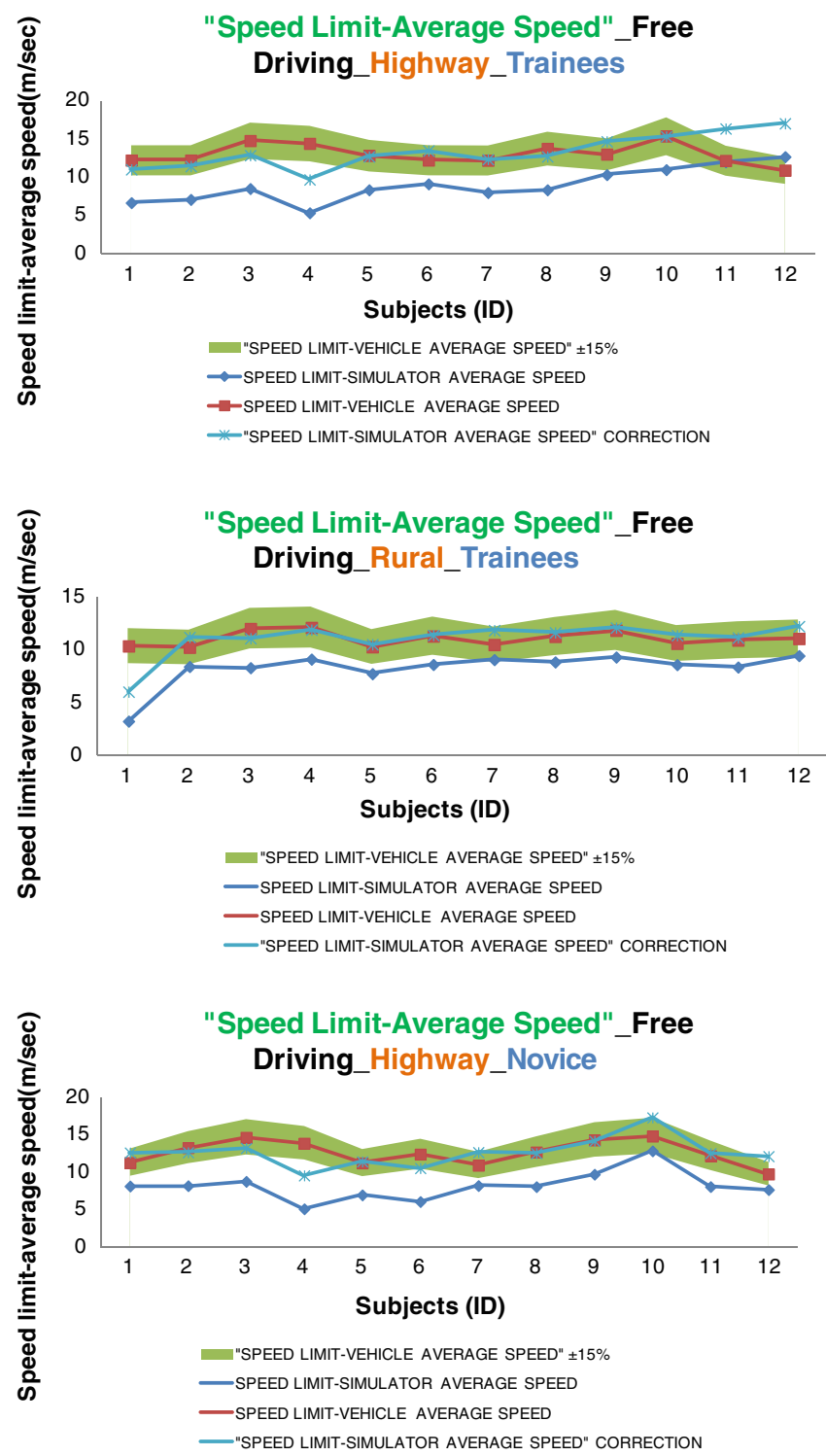

- In the case of experienced divers in rural and urban roads, there is no numerical proximity being observed, with or without correction (Correlation Type D-E), while that relative numerical proximity occurs with no need for correction (Correlation Type B) is in the case of trainees in urban roads (Fig. 5). We observe that, by default, the more the driving experience increases, the more the unrealism of the driving simulator is being observed and the driving behaviour in it is changing.

- In each case, when a correction was required (Correlation Type $C$ ), the numerical values were bigger in the real traffic conditions from those in driving simulator. However, we should underline, for the avoidance of any confusion, that in this case, the higher the values are, the lower the average speed is, which means that in the driving simulator, the drivers maintained
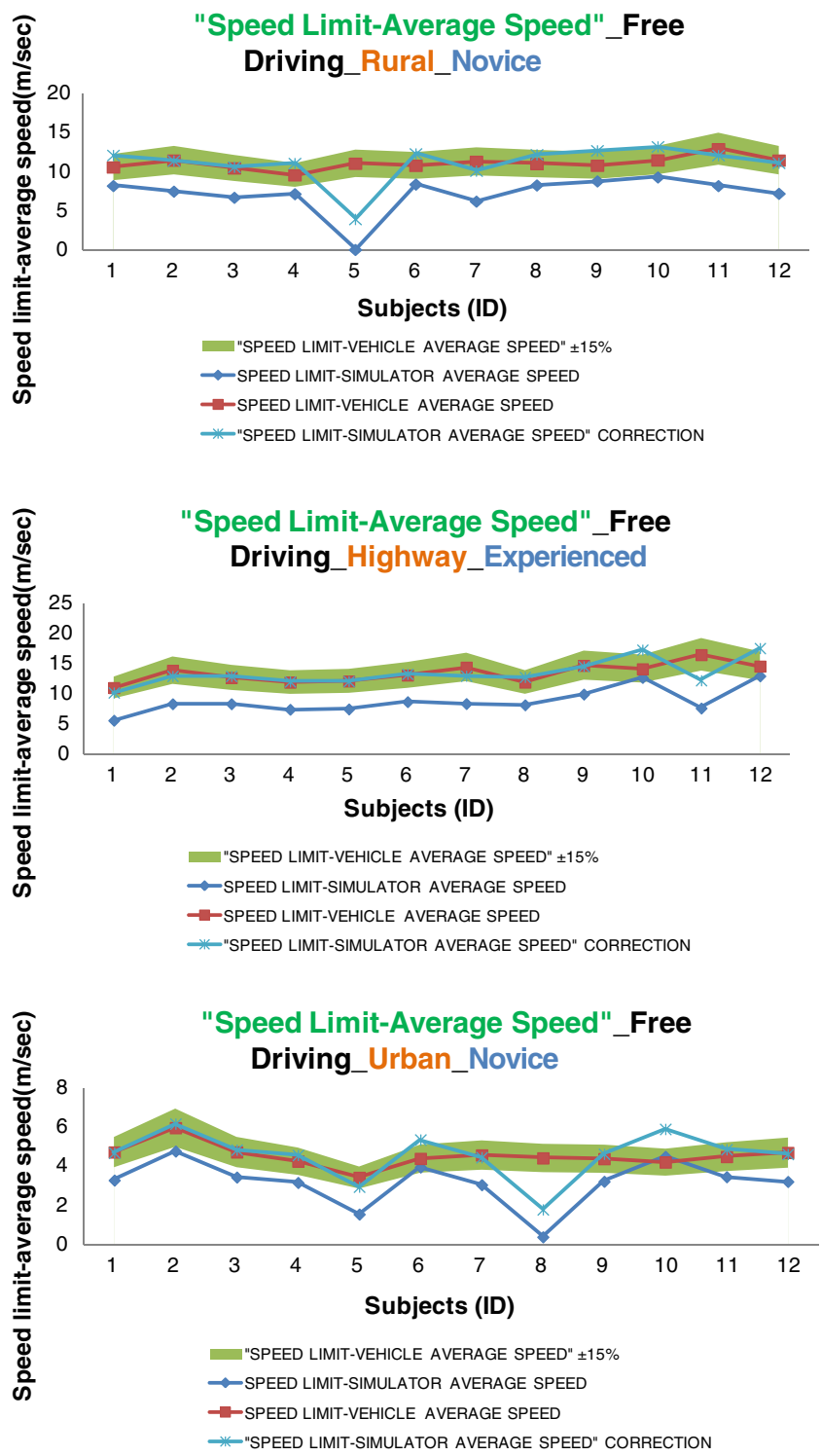

Fig. 4 Difference of average speed from speed limit results corresponding to Correlation Type C 


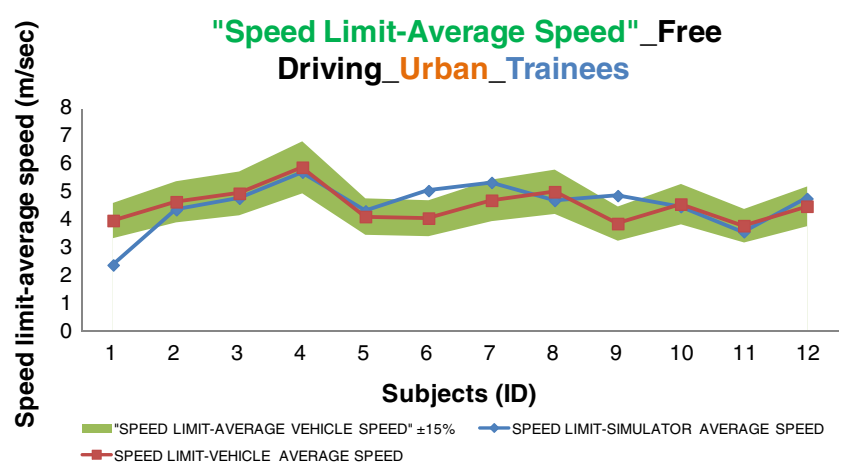

Fig. 5 Difference of average speed from speed limit results corresponding to Correlation Type B

\section{bigger average speed than in reality, and for this reason they approached the speed limit more each time.}

The matrix of Eq. 7 of the previous section summarises the corresponding transfer algorithms related to the difference of the average speed from the speed limits.

\subsubsection{Average Lateral Deviation}

As aforementioned, lateral deviation in this case is meant to be the distance of the driver's vehicle from the middle of his/her traffic lane. It should be mentioned that lateral deviation was recorded only in the case that an indicator-expressing wish to change lanes or execute deliberate manoeuvres- was not used by the driver.

The average lateral deviation has been analysed only for highways, since in the other road contexts, the limits of the lane are not clear in real traffic conditions in the majority of the Greek roads, which does not allow valid correlation and analysis of the recorded measurements.

The major remarks concerning the average lateral deviation from the middle to the left of the lane are the following:

- In this case, numerical proximity does not emerge in any case even after correction (in any scenario or road context). As such, there is no transfer algorithm occurring for average lateral deviation (Figs 6 and 7).

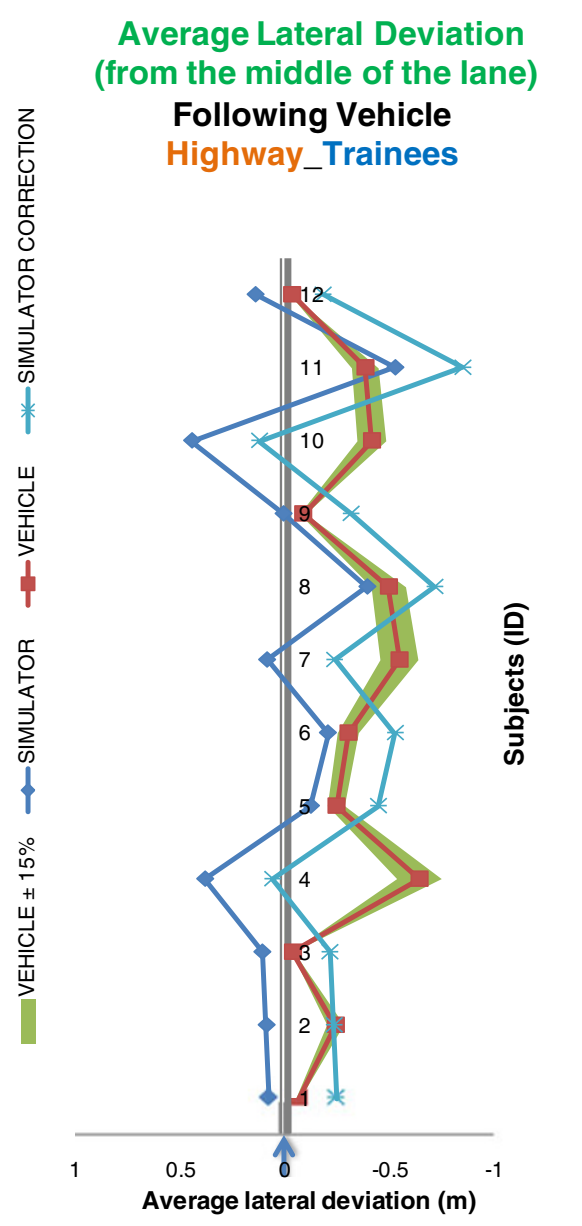

Lane middle
Average Lateral Deviation (from the middle of the lane)

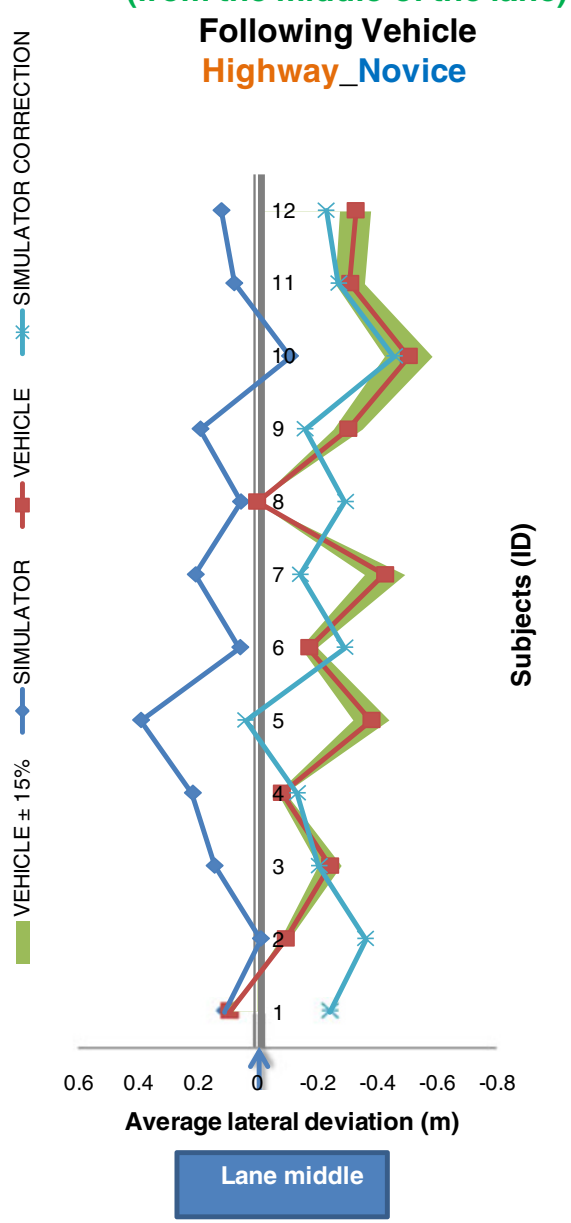

Average Lateral Deviation (from the middle od the lane) Following Vehicle Highway_Experienced

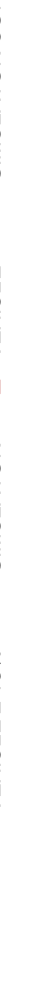

0.6

Average lateral deviation (m)

Lane middle

Fig. 6 Average lateral deviation in following vehicle scenario 
- However, in the free driving scenario, drivers seem to be clustered in two groups (in all driver groups, regardless of their driving experience). In a cluster that presents relatively similar driving behaviour in real traffic conditions and in driving simulator, and in another group, the driving behaviour of which in the two contexts is totally different (see Fig. 7 respectively). This may due to the similar predictive model of some drivers or due to the fact that some drivers did not confront with the same responsibility the driving sessions in the simulator, and, as such, there is a significant deviation from their driving behaviour on road, and this does not seem to be related to their driving experience or the driving context. The second case is not likely to occur in processes where simulator driving sessions constitute part of the driving assessment for the acquisition (or renewal) of a driving license. In such a case, it would be meaningful to compare again the driving behaviour on road and in driving simulator. In other caseswhere the driver does not have a direct benefit from his/her driving in the simulator, it would be perhaps effective if a kind of penalty could be applied in order to avoid the depreciation of the driving in simulators. Nevertheless and concerning the first possible reason for this behaviour being observed-predictive models effect-this observation could serve as a starting point for further analysis in the field of cognitive models and their effects in simulator driving.

- In all cases, in both scenarios (free driving and following vehicle), it was observed that drivers tended to drive towards the right edge of the lane more often in the real traffic conditions than in the driving simulator.

- However, in the free driving scenario, a displacement of the drivers' average lateral deviation towards the left edge of the traffic lane is being observed, both in the driving simulator and in real traffic conditions. As a result, drivers approach more the middle of the lane in real traffic conditions.

- What can be generally concluded from the results concerning the average lateral deviation is that drivers seem not to be really convinced from the riskiness that is being reflected in the driving simulator, and perhaps this is the reason that, in the simulator, they tend to drive more to the left than in real traffic conditions. In addition, in the free driving scenarios, as they do not have to execute a concrete driving task, they tend to drive more
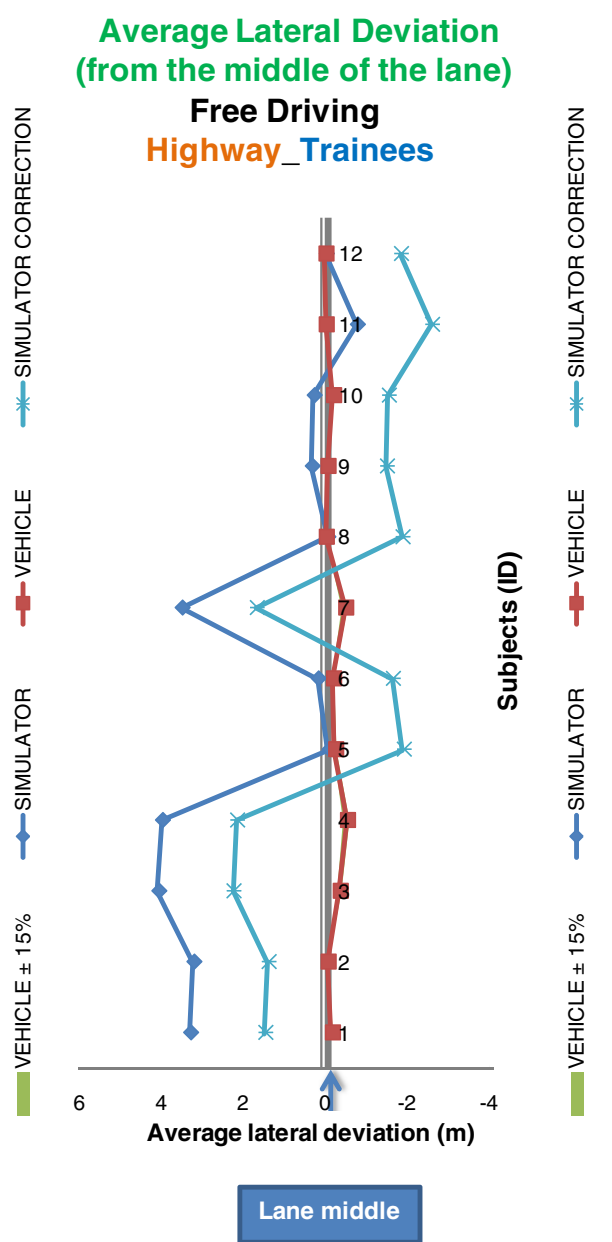

Average Lateral Deviation
(from the middle of the lane)

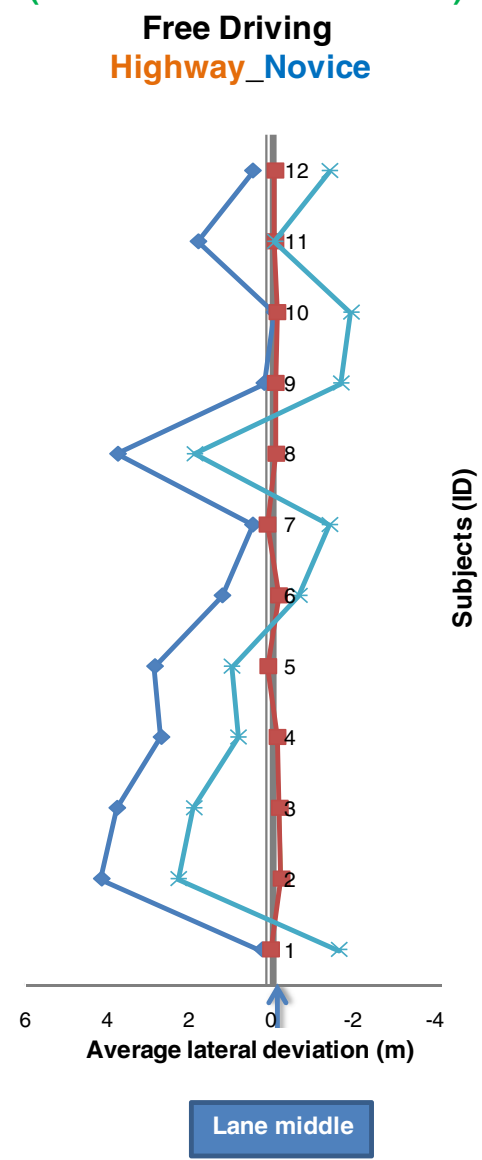

Average Lateral Deviation (from the middle of the lane)

Free Driving

Highway_Experienced

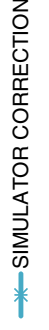

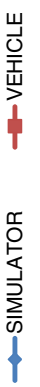
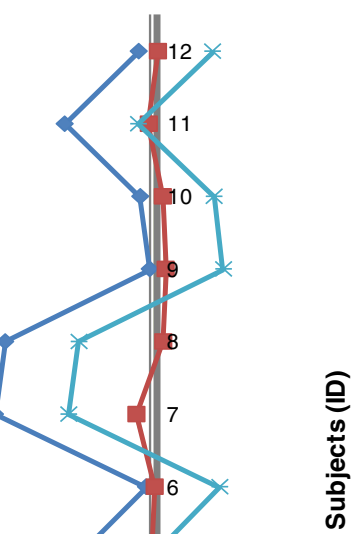

Fig. 7 Average lateral deviation in free driving scenario 
to the left of the lane, most likely in order to execute other maneuvers (i.e. check for overtaking, etc.).

In the above figures, the positive values denote displacement towards the left of the lane and negative values denote displacement towards the right of the lane.

\section{Conclusions and Further Research}

This manuscript presents the experimental plan and the results of driver trials being conducted with a semi-dynamic driving simulator and a specially equipped research vehicle in real traffic conditions. 12 trainees, 12 novice and 12 experienced drivers participated in the trials, running in both simulator and on-road, the same driving tasks, namely free driving and following vehicle, in three different road contexts in each, namely highways, rural and urban roads. Their driving performance regarding maximum speed, difference of average speed from speed limit and average lateral deviation was collected from the driving sessions in simulator and on-road and a correlation study followed with respect to the investigation of the numerical proximity of the results in the two contexts, in the sense of absolute validity.

For the sake of the analysis, a novel method was established. 4 Correlation Types have been defined for the analysis of the results, each one corresponding to each of the four research hypotheses being originally developed and addressed in this manuscript (out of the 6 in total). They are dealing with the existence or not of numerical proximity between the driving simulator measurements and those corresponding to real traffic conditions (in the sense of absolute validity), the degree of this proximity, and whether a correction is applicable-and which that would be- in order to reach it.

The first research hypothesis corresponds to the results that fulfill the so-called Correlation Types A and B, which assume an absolute or relative numerical proximity of the measurement respectively, with no need for further correction. For the driving behaviour metrics being discussed in this manuscript, results show that Correlation Type A (absolute numerical proximity) is not reached in any case. However, relative numerical proximity (Correlation Type B) has been resulted in the maximum speed of trainees and experienced drivers in highways and urban roads, as well as in the different of the average speed from the speed limit of the trainees in urban road contexts, all in free driving conditions.

The above results indicate in which cases, it would be safe to conduct trials in semi-dynamic driving simulator with no further need for verification in real traffic conditions. However, due to the fact that the Correlation Type being fulfilled is $\mathrm{B}$ and not $\mathrm{A}$ (which means that the result is valid for at least $75 \%$ of the driver population), any extrapolation of the results to the general population should be done with some reservation. As such, research hypothesis 1 can be partially accepted, for some driving behaviour metrics, some driving tasks/scenarios and some drivers cohorts for the specific type of semi-dynamic driving simulator.

The second research hypothesis corresponds to the results that fulfill the so-called Correlation Type C (relative numerical proximity after correction following the linear model). As it has been shown, many more cases apply here than in the previous type. These cases are the maximum speed of novice drivers in highways and urban roads, in the maximum speed of trainees in rural roads in the difference of average speed from the speed limit of novice and experienced drivers in highways, of trainees and novice drivers in rural roads and of novice drivers in urban roads, all in the free driving scenario.

These results indicate that it would be safe to conduct trials in semi-dynamic driving simulator, as long as the necessary corrections are put in place. As such, research hypothesis 2 can be also partially accepted for some driving behaviour metrics, some driving tasks/scenarios and some drivers cohorts for the specific type of semidynamic driving simulator.

As it has been discussed in the current manuscript, the third research hypothesis has been rejected for the specific type of semi-dynamic simulator, since it has been proved that even if there is a sufficiently strong non-linear correlation leading to an acceptable numerical proximity, this is especially sensitive to the subjects sample, and as such, it is considered a rather unsafe method for corrections that could be extrapolated to the general population.

The fifth research hypothesis matches to the results fulfilling Correlation Type E (and finally D-E) which assumes no correlation that may lead to acceptable numerical proximity. Indeed, there are several cases that this is valid, and as such, the fourth research hypothesis is partially accepted for the specific type of semi-dynamic driving simulator.

As the results have shown, it seems that what matters the most, concerning the absolute validity of this specific type of driving simulator, is the type of the driving behaviour metric. This is the factor that, most of all, defines the existence and the level of the numerical proximity between the driving simulator and the real traffic conditions measurements. The effect of other parameters, like the driving experience, the type of the driving task or the road context require further investigation.

It should be reminded that absolute validity in this analysis is being investigated with a set tolerance threshold of $15 \%$ (absolute value), which relates with the measurement errors of the research vehicle. This threshold may likely differ depending on the research vehicle that participates in the trials. Consequently, if other thresholds would be applied, the results would be interpreted in a different way. 
In addition, the indicative results being presented in this manuscript concern the specific type of semi-dynamic driving simulator being used. Further research in this field would address other types of simulators (i.e. dynamic, static).

As aforementioned, the use of linear and non-linear regression for the determination of the required correction has been rejected as an unsafe method in this case. However, a bigger sample might likely lead to different results and would allow the investigation of the statistical significance of the results, which with the current sample size was not possible. In specific, in order to apply a one-sample $t$-test (one-tailed), which would be applicable here, at least 27 persons per comparison (and consequently per driver group) would be required (for a power of 0,8 and Cohen medium effect size of 0,50 ). Definitely, priority for more trials with bigger sample should be given to the cases that already present a strong tendency, meaning those that have resulted in transfer algorithms.

In addition, further research would be interesting to encompass other driver cohorts (i.e. elderly drivers), since from the current trials did not reveal any clear differentiation between the driver cohorts that were included, despite our original expectations, although we should be skeptical on how this result would differ if the trials would be conducted with bigger samples.

Also, the lateral deviation results have revealed a correlation weakness with respect to driving performance related with the lateral axis of the road, most probably due to the insufficient dynamics of the driving simulator in this respect, though this should not hinder further investigation in this area.

The derived results apply in various research fields, with ADAS research and driving skills training and assessment being most likely the key ones. The findings, the most tangible of which are being summarised in the emerging transfer algorithms, may serve as the basis for redefining warning and intervention thresholds of ADAS but also driver training and assessment schemes (encompassing driving simulator), which are often erroneously developed. The transfer algorithms being presented in the respective matrices for each metric, which may be even broader in near future, addressing more types of driving simulators, driving behaviour metrics, driving tasks, road contexts and driver cohorts, may support decision-making regarding the worthwhile investments in driving simulator applications and the cost-effective use of the significantly more costly trials in real traffic conditions, since they denote when driving simulator may safely replace real world. In addition, costeffectiveness of this approach lies in the fact that applying such a method, valid results concerning driving behaviour and in projection road safety, are not dependent of the availability of high cost and fidelity driving simulators.

Last but not least, this approach is based on examining the behaviour of each driver individually and, not statistically, in the context of a general population. The validity of this approach has been verified in several cases, where results have shown that the cognitive and perceptual model and the psychomotor characteristics of each individual are in some cases beyond other characteristics that usually serve for the creation of driver cohorts and possibly explains why in some cases, driving behaviour in driving simulator and in real traffic conditions cannot be deterministically related.

Open Access This article is distributed under the terms of the Creative Commons Attribution License which permits any use, distribution, and reproduction in any medium, provided the original author(s) and the source are credited.

\section{References}

1. Blaauw GJ (1982) Driving experience and task demands in simulator and instrumented car: a validation study. Hum Factors 24(4):473-486

2. Jamson H (1999) Curve negotiation in the Leeds driving simulator: The role of driver experience. In: Harris D (ed) Engineering in psychology and cognitive ergonomics, Vol. 3. Ashgate, Aldershot, pp 351-358

3. Kaptein NA, Theeuwes J, Van der Horst R (1995) Driving simulator validity: Some considerations. Pre-print of a paper presented at the TRB 75th Annual Meeting

4. Blana E (1996) Driving simulator validation studies: A literature review. Institute of Transport Studies, University of Leeds, Working Paper 480

5. Godley ST, Triggs TJ, Fildes BN (2002) Driving simulator validation for speed research. Accid Anal Prev 34:589-600

6. Blana E, Golias J (2002) Differences between vehicle lateral displacement on the road and in a fixed-base simulator. Hum Factors $\mathrm{J}$ Human Factors Ergon Soc 44(2):303-313

7. Lee HC (2003) The validity of driving simulator to measure onroad driving performance of older drivers. Transp Eng Aust 8:89100

8. Hirata T, Yai T, Takagawa T (2007) Development of the driving simulation system MOVIC-T4 and its validation using field driving data. Tsinghua Sci Technol 12(2):141-150

9. De Winter JCF, De Groot S, Mulder M, Wieringa PA, Dankelman J, Mulder JA (2009) Relationships between driving simulator performance and driving test results. Ergonomics 52(2):137-153

10. Riener A (2010) Assessment of simulator fidelity and validity in simulator and on-the-road studies. Int J Adv Syst Meas 3(3\&4):110 124

11. Bella F (2008) Driving simulator for speed research on two-lane rural roads. Accid Anal Prev 40:1078-1087

12. Lee HC, Cameron D, Lee AH (2004) Assessing the driving performance of older adult drivers: on-road versus simulated driving. Accid Anal Prev 35:797-803

13. Yan X, Abdel-Aty M, Radwan E, Wang X, Chilakapati P (2008) Validating a driving simulator using surrogate safety measures. Accid Anal Prev 40:274-288

14. Lew HL, Poole JH, Lee EH, Jaffe DL, Huang HC, Brodd E (2005) Predictive validity of driving-simulator assessments following traumatic brain injury: a preliminary study. Brain Inj 19:177-188

15. Patomella A-H, Tham K, Kottorp A (2006) P-drive: assessment of driving performance after stroke. J Rehabil Med 38:273-279

16. Lee HC, Lee AH (2005) Identifying older drivers at risk of traffic violations by using a driving simulator: a 3-year longitudinal study. Am J Occup Ther 59:97-100 
17. Fujioka T, Muramatsu K (1996) Drivers' behavior in ITS environment investigated by a driving simulator. In Proceedings of IEEE Intelligent Vehicles Symposium, pp 295-299

18. Liao D (2006) A real-time high-fidelity driving simulator system based on pc clusters. In: Proceedings of 11th IEEE International Conference on Engineering of Complex Computer Systems (ICECCS), Stanford, California

19. Yu S, Lee S, Kim S, Lee D (2006) Development and evaluation of ITS devices using KAAS system. In: Proceedings of Society of Instrument and Control Engineers (SICE)/Institute of Control, Automation and Systems Engineers (ICASE) International Joint Conference, Busan, pp 2116-2120

20. Kim KD, Kim MS, Moon YG, Lee MC (2006) Application of vehicle driving simulator using new washout algorithm and robust control. In: Proceedings of Society of Instrument and Control Engineers (SICE)/ Institute of Control, Automation and Systems Engineers (ICASE) International Joint Conference, Busan, pp 2121-2126

21. Miyajima C, Nishiwaki Y, Ozawa K, Wakita T, Itou K, Takeda K (2006) Cepstral analysis of driving behavioral signals for driver identification. In: Proceedings of IEEE International Conference on Acoustics, Speech and Signal, Vol. 5, pp 921-924

22. Kendall IR, Jones RP (1999) An investigation into the use of hardware-in-the-loop simulation testing for automotive electronic control systems. Control Eng Pract 7(11):1343-1356

23. McGehee DV, Brown TL, Lee JD, Wilson TB (2002) The effect of warning timing on collision avoidance behavior in a stationary lead-vehicle scenario. Transp Res Rec 1803:1-7

24. Evans L (ed) (1991) Traffic safety and the driver. Van Nostrand Reinhold (Publ.), New York

25. Kappé B, Emmerik ML (2005) The use of driving simulators for initial driver training and testing. Report: TNO Defence, Security and Safety, 75151.01

26. Vlakveld WP (2005) The use of simulators in basic driver training, HUMANIST Workshop, Brno

27. De Winter JCF, Wieringa PA, Dankelman J, Mulder M, Van Paassen MM, De Groot S (2007) Driving simulator fidelity and training effectiveness. In: Proceedings of the 26th European Annual Conference on Human Decision Making and Manual Control, Lyngby, Denmark

28. Dorn L (2008) Is it the real thing? adiNEWS, Psycho, pp18-19. http://www.drivermetrics.com/

29. Bekiaris E, Panou M, Tsioutras A (2007) The usage of modern tools and methods for drivers' training", Thessaloniki, Greece 2007 , ISBN 978-960-930008-7

30. Evans L (2004) Traffic safety. Science Serving Society, Bloomfield Hills

31. Brouwer WH, Waterpink W, Van Wolfelaar PC, Rothengatter T (1991) Divided attention in experienced young and older drivers: lane tracking and visual analysis in a dynamic driving simulator. Hum Factors 33:573-582

32. Stelmach GE, Nahom A (1992) Cognitive-motor abilities of the elderly driver. Hum Factors 34:53-65
33. Reed MP, Green PA (1999) Comparison of driving performance onroad and in a low-cost simulator using a concurrent telephone dialing task. Ergonomics 42(8):1015-1037

34. Verwey WB (2000) On-line driver workload estimation. Effects of road situation and age on secondary task measures. Ergonomics 43:187-209

35. Aronsson K, Bang KL (2006) Female and male driving behaviour on Swedish urban roads and streets. In: European Transport Conference (ETC)

36. Carsten OMJ, Tight MR, Southwell MT, Plows B (1989) Urban accidents: Why do they happen? Basingstoke: AA Foundation for Road Safety Research, UK

37. Minderhoud MM, Bovy PHL (2001) Extended time-to-collision measures for road traffic safety assessment. Accid Anal Prev 33:89-97

38. Hiramatsu M, Obara H (2000) Rear-end Collision scenarios categorized by type of human error. Society of Automotive Engineers of Japan, Review 21, Elsevier, Japan, pp 535-541

39. Archer J (2005) Indicators for traffic safety assessment and prediction and their application in micro-simulation modelling: A study of urban and suburban intersections. Doctoral dissertation, Karlstad University, Karlstad, Sweden

40. Östlund J, Björn P, Thorslund B, Engström J, Markkula G, Keinath A, Regienov DH, Mattes S, Foehl U (2006) Driving performance assessment-methods and metrics. Deliverable 2.2.5, AIDE Integrated Project, Contract No: IST-1-507674-IP

41. Vogel K (2003) Modelling driver behaviour: A control theory based approach. Doctoral dissertation, No. 751, Institute of Technology, University of Linköping, Linköping Studies in Science and Technology, Linköping, Sweden

42. Peters B, Östlund J (2005) AIDE VTI WP2.2 vehicle sensor requirements. AIDE Internal deliverable, AIDE Integrated Project, Contract No: IST-1-507674-IP

43. Kennedy RS, Lane NE, Berbaum KS, Lilienthal MG (1993) Simulator sickness questionnaire: an enhanced method for quantifying simulator sickness. Int J Aviat Psychol 3(3):203-220

44. Zijlstra FRH (1993) Efficiency in work behavior. A design approach for modern tools. Doctoral dissertation, Delft University of Technology, Delft, the Netherlands: Delft University Press

45. Brookhuis KA, DeVries G, Prins Van Wijngaarden P, Veenstra G, Hommes M, Louwerens JW, O'Hanlon JF (1985) The effects of increasing doses of Meptazisol (100, 200, $400 \mathrm{mg}$ ) and Glaferine (200mg) on driving performance. Report VK 85-16. Haren, The Nethrlands: Traffic Research Center, University of Groningen

46. He M, Liu X, Rong J (2002) Driver's preferred time headway selection: Experimental findings. In: Proceedings of 3rd International Conference on Traffic and Transportation Studies. Reston, VA: ASCE

47. SAVE Consortium (1997) Methods and criteria for detection of driver impairment. Deliverable 5.1, SAVE Project, Contract No: TR 1047 